\title{
Ira actual depresión internacional y los países en vías de desarrollo
}

1. La actual depresión de alcance mundial -sin duda alguna grave- tiene características propias y puede distinguirse de la de los años treinta en muchos aspectos. Pero también tiene diferentes consecuencias para los países en desarrollo y para la economía mundial como un todo. Su repercusión en los países en desarrollo necesita estudiarse con gran cuidado, así como deben también examinarse los posibles efectos positivos que puedan provenir de la probable recuperación de la economía norteamericana y, a continuación, de las economías de otros países desarrollados, principalmente Japón, la República Federal Alemana, Francia y Ios Países Bajos. En este análisis no deberán pasarse por alto los problemas fundamentales de los países del Tercer Mundo.

2. Las principales diferencias entre la actual recesión/depresión y la de los años treinta pueden clasificarse en cinco categorías de problemas:

a) Los países industrializados de Occidente se encuentran enfrascados en profundos problemas estructurales que resultan de tasas diferenciales de cambio técnico entre ramas industriales o dentro de las mismas ramas, y entre países (por ejemplo, entre Estados Unidos y Japón, o entre Europa Occidental y los demás). Determinadas industrias empiezan a ser obsoletas en algunos de los países; por ejemplo, la industria siderúrgica de los Estados Unidos y de partes de Europa. Destaca también el impacto que las aplicaciones japonesas de los procesos microelectrónicos y de la robotización están teniendo sobre Estados Unidos y algunos países europeos. La calidad de los automóviles japoneses es otro factor importante. Además, la ampliación de los programas de bienestar social y su efecto sobre el ingreso real, y especialmente sobre el salario real, han influido en la incidencia del sector público sobre el producto bruto a través de la creciente proporción del gasto que debe asignarse a todos los aspectos del bienestar social, incluido desde luego el seguro contra el desempleo en sus varias formas, el seguro médi$\mathrm{co}$, etc.

Por otra parte, los países desarrollados están en su mayoría em. 
peñados en una carrera de armamentos de graves consecuencias en sus presupuestos $y$, en consecuencia, en los déficit presupuestarios. Es también una carrera armamentista de naturaleza altamente técnica que no necesariamente se traduce en ampliación de los efectivos de las fuerzas armadas y de las disponibilidades de equipo corriente, sino que más bien representa un desplazamiento hacia equipos de gran refinamiento: proyectiles, ojivas nucleares, aviones $y$ tanques de gran costo, etc.

Todos estos problemas quizás puedan clasificarse bajo el término "problemas estructurales".

b) Los países en vía de desarrollo, que a su vez deben clasificarse según su grado de desarrollo y la naturaleza de su propio cambio estructural están en una situación distinta a la que tenían en los años treinta. Existe gran diferenciación entre los países en desarrollo como un todo. Los de nueva industrialización no pueden compararse con aquellos que continúan siendo predominantemente agrícolas $y$, como referencia de desarrollo, relativamente primitivos, como lo son muchos de Africa y Asia. Los paises de nueva industrialización afrontan problemas especiales derivados de sus formas de industrialización: políticas de sustitución de importaciones que han resultado ser de gran costo real - justificadas no sólo por motivos económicos sino por otros- y llevadas a cabo con apoyo en elevada protección arancelaria y otras formas de restricciones. Una gran proporción de estas nuevas industrias no es competitiva internacionalmente; sin embargo, es indudable que han creado empleo.

En Ios últimos cuarenta a cincuenta años, el crecimiento demográfico de la mayor parte de los países en desarrollo -esto es, la tasa de natalidad relacionada con la tasa de mortalidad- ha sido más rápido que en la mayor parte de los demás países en cualquier tiempo anterior de la historia. Esto se ha debido principalmente al descenso de la mortalidad, en tanto que las tasas de fecundidad han permanecido relativamente estables, o en algunos casos inclusive se han elevado. Hay indicios de que en. los últimos diez años, la natalidad ha descendido en algunos países en desarrollo, entre ellos en unos cuantos países de América Latina y de Asia, El caso de Ghina es de carácter especial, pero también registra descensos del crecimiento de la población. En todo caso, el amplio aumento de la población y sus características, es decir, a base de alta tasa de natalidad con baja tasa de mortalidad, ha significado un ritmo de incremento muy rápido de la población en edades jóvenes y de la población en edad de trabajo, y en consecuencia de la fuerza de trabajo. En algunos casos esta última se ha incrementado aún más rápidamente debido a la mayor proporción de participación de las mujeres en el trabajo remunerado, como reșultado de diversos factores sociales y culturales.

El crecimiento rápido de la población no sólo significa que los 
esfuerzos por incrementar el empleo han tenido que ser más intensos, sino también que en realidad apenas en unos cuantos casos el empleo se ha incrementado a un ritmo y en una forma que pudiera reducir sustancialmente el subempleo preexistente. La industrialización ha ampliado, desde luego, las oportunidades de empleo y ha modificado la naturaleza y la estructura de éste, pero se ha basado principalmente en tecnologías ahorradoras de trabajo -forma de empleo inferior al óptimo social. Subsiste, por lo tanto, un grave problema de interacción negativa entre crecimiento demográfico rápido e industrialización moderna. Pocos países han podido escoger un camino - quizás China sea la excepción- que incluya formas de industrialización y de creación de otras actividades que absorban la creciente fuerza de trabajo en medida adecuada.

Debiera advertirse que los países desarrollados han llegado a una situación de crecimiento demográfico muy lento, o aún negativo, al grado de que debido a sus problemas industriales y a sus estructuras salariales han tenido que importar de los países en desarrollo, ya sea temporalmente -0 , poco a poco, sobre base permanente- una parte de su fuerza de trabajo. Esto ha ocurrido en Europa, así como en Estados Unidos. Entre ciertos países en desarrollo, incluidos los productores de petróleo del Oriente Medio, se ha registrado también bastante migración diferencial dirigida a mercados de trabajo segmentados.

Se ha modificado la estructura mundial de la población, con mucho mayor proporción de la población en los países en desarrollo. La mayor parte, con mucho, del incremento demográfico mundial ocurrirá en estos mismos países.

Otro aspecto de los cambios en los países en desarrollo es la persistencia de los probleanas de desarrollo rural. Con pocas excepciones, estos países se han vuelto importadores netos de alimentos, si bien al mismo tiempo poseen recursos disponibles, $y$, al menos en teoría, podrían en muchos casos alcanzar un alto grado de autosuficiencia. Las causas de la insuficiente producción agropecuaria $y$ del lento desarrollo rural son muchas, algunas de ellas institucionaIes y culturales a la vez que sociales, y otras resultan de las estructuras de los precios relativos. Sin embargo, no hay duda de que, en muchos casos, el crecimiento demográfico mismo y la transformación de la estructura industrial y urbana de los países han generado cambios en la composición del consumo de alimentos a los cuales no han podido responder adecuadamente los factores de oferta en las áreas rurales.

Ha surgido además un cambio estructural que se ha acentuado en los últimos diez años, y que es la dependencia energética de gran número de países en desarrollo. Su industrialización se ha llevado a cabo en su mayor parte sobre la base de energia barata, sea la desarrollada nacionalmente o la importada, y se han establecido mo- 
dalidades tecnológicas en las que la sustitución energética-ahora que la energía se há vuelto más cara internacionalmente- no ha podido llevarse a cabo con facilidad. En lo general, en los países subdesarrollados no existen alternativas tecnológicas importantes a la dependencia energética. Desde Iuego que esta dependencia es también característica de numerosos países altamente industrializados, pero muchos de ellos han podido, debido a su capacidad tecnológica, economizar energía por unidad de producto industrial, o en la rama de consumo de sus economías. Han podido también desarrollar alternativas, por ejemplo pasar del petróleo al gas y al car. bón, o de los hidrocarburos a otras fuentes energeticas, notablemente la energía nuclear, $y$, aunque todavía en pequeñas proporciones, a las energías no convencionales. Está pues ocurriendo un cambio a escala mundial en la estructura de la oferta y del consumo ener. géticos, siendo más rápido el crecimiento del consumo en los países en desarrollo. Todo ello ha afectado los diversos aspectos de las relaciones económicas internacionales, las estructuras de inversión, las necesidades de tecnología, y aún las políticas económicas y las relaciones políticas.

Los países en desarrollo son también mucho más dependientes desde el punto de vista financiero de lo que fueron en los años treinta. Algunos han experimentado mejoría en su relación de precios del intercambio o han desarrollado nuevas fuentes de divisas a través de la exportación de manufacturas - de lo cual algunos países se han beneficiado considerablemente y a gran ritmo- Sin embargo, debido a diversas circunstancias, en especial las relacionadas con el auge petrolero en el Oriente Medio y con la vasta creación de petrodólares, numerosos países en desarrollo han tenido también acceso al crédito externo múcho más allá de las disponibilidades de los organismos financieros multilaterales y regionales; en otras palabras, han tenido acceso a los sistemas de banca comercial a través del reciclaje de los petrodólares y de otros instrumentos de liquidez creados en Europa y en los Estados Unidos en los últimos cinco a diez años. Los ritmos de incremento del endeudamiento externo en los países en desarrollo han llegado a ser tales que su deuda total alcanza los 600 mil millones de dólares, mucha de ella a corto plazo y a altas tasas de interés, concentrada en unos cuantos países de nueva industrialización. Debido a la rigidez a corto plazo de los pagos de servicio de la deuda (incluidos los intereses), se han vuelto especialmente vulnerables las situaciones de balanza de pagos con relación a las fluctuaciones recientes de precios de los productos básicos y a las políticas proteccionistas que siguen los países desarrollados respecto a las importaciones de manufacturas de los paises en desarrollo. Las fluctuaciones económicas generales, sobre todo la recesión de los últimos años, han tenido un efecto acentuado en el Tercer Mundo. Durante los años treinta hubo dependencia 
financiera, y la mayor parte de los países en desarrollo estaba de hecho en situación de moratoria de deuda, pero no parece ser que su dependencia fuera tan grande como parece ser hoy día, simplemente desde el punto de vista de mantener en funcionamiento sus economias.

Los factores anteriores no son todos negativos -algunos en realidad son positivos- pero en épocas de recesión las rigideces esenciales y los factores negativos sobresalen y pueden no ser fácilmente objeto de compensación.

c) Los países socialistas -economías de planificación central-, en especial los de Europa Oriental, comparten muchos de los problemas tanto de los países desarrollados de Occidente como de los países en desarrollo. Esto es, mantienen industrias obsoletas, intervienen en una fuerte carrera armamentista, no han podido mantenerse al corriente en innovación tecnológica, y por otro lado, con la excepción de la Unión Soviética, son países dependientes de importaciones dẹ energéticos, y casi todos se han vuelto dependientes financieramente y se han entrelazado muy sutilmente con el resto de la economía mundial, especialmente con los países industrializa. dos de economía de mercado. Además, algunos, no obstante su lento crecimiento demográfico, han fracasado en sus políticas agrícolas y han pasado a ser importadores netos de alimentos. Por supuesto que el bienestar social es una política obligatoria en los países socialistas, pero la calidad y los niveles de dichos programas sociales no son siempre muy altos, y en general el consumo se ha sacrificado a la inversión y al gasto militar. Ha habido mejoras, pero no puede decirse, sino con excepciones, que el consumo corriente en los países socialistas corresponda a sus posibilidades tecnológicas $y$ a las aspiraciones de los pueblos de esos países.

d) Una de las características de la actual depresión mundial es que la inflación está presente en los tres grupos de países. En los países industrializados la inflación se ha reducido a expensas de un vasto desempleo y de una suspensión de nuevas inversiones en la industria en general, y aún en la agricultura. En algunos países de Europa Occidental la inflación continúa siendo bastante elevada. La política de corto plazo o coyuntural se ha llevado muy violentamente hacia el control de la inflación por medios monetarios, y esto a su vez ha hecho más difícil llevar a cabo muchos de los cambios estructurales necesarios.

En los países en desarrollo, las tasas de inflación, sobre todo en América Latina, han sido sumamente elevadas, o aún se han incrementado. Uno o dọs países han aprendido a "vivir con la inflación". Ha habido sucesivas crisis de balanza de pagos, devaiuaciones, resurgimientos de la inflación y nuevas devaluaciones, a veces seguidas de estancamiento de la economía, sin haberse logrado reducir mucho la tasa de inflación. Los factores estructurales expli- 
cán en parte esta situación, como también en algunos países la explican los cambios en los precios internacionales relativos, sobre todo el efecto negativo del incremento de los precios del petróleo en los últimos diez años. En otros, sobre todo en los países exportadores de petróleo (por ejemplo, México y Nigeria), la misma exportación ide petróleo en tan grandes cantidades, a precios crecientes, ha sido un factor de inflación a través de su impacto en la demanda interna tanto de inversión como de consumo. Esto es especialmente cierto en aquellos países que no han podido cerrar la brecha cle ahorro interno a través de políticas fiscales y financieras adecuadas.

En las economías de planificación central puede decirse también que ha surgido la inflación, en gran parte ocultada por el sistema de control de precios y de distribución no mercantil de productos tanto para producción como para consumo. Los resultados de estas presiones sobre el sistema y de los desequilibrios se han reflejado con frecuencia en situaciones de escasez, equivalentes a inflación y en muchos casos ha sido necesario reajustar los precios.

e) La recesión/depresión actual ha sido en parte inducida por los esfuerzos por controlar la inflación a través de políticas monetarias radicales, sin cambio en la estructura del gasto en armamentos y con rigidez en los gastos en bienestar social. Sin embargo, también se ha producido un descenso, en los países altamente industrializados y en las economías de planificación central de Europa Oriental, de la tasa de crecimiento de la productividad. En los países de economía de mercado occidentales se han aumentado además constantemente los salarios reales, y en algunos casos la jornada semanal de trabajo ha descendido. Toda esta es una combinación inusitadamente desfavorable de tendencias, que también distingue a la actual depresión de la de los años treinta y hace más difícil para el mundo en general salir de ella.

En general, se han transformado considerablemente las corrientes comerciales. En los años treinta, los países industriales importaban en lo esencial materias primas y algunos alimentos, y exportaban bienes de consumo manufacturados, productos intermedios y algunos bienes de capital a los países en desarrollo. Entre ellos intercambiaban alimentos y minerales, así camo productos energéticos, y gran número de productos manufacturados. Los años treinta fueron una época de elevada protección arancelaria y administrativa en la que se introdujeron controles y contingentes de importación. Fue también una época de inestabilidad monetaria y de restricciones al comercio, resultantes de las políticas de corto plazo o del funcionamiento automático del patrón de cambio oro a tipos de cambio sobrevaluados. En la actualidad, en medio de la gran depresión de hoy, la proporción del comercio mundial que se lleva a cabo entre los paises industrializados y entre países occidentales 
y socialistas, respecto a casi cualquier clase de manufactura y servicio, ha aumentado considerablemente. Al mismo tielmpo, los paises en desarrollo se han vuelto exportadores de manufacturas, no sólo de sencillos bienes de consumo, sino también de ciertas clases de productos intermedios y de bienes de capital. Este comercio se ha dirigido en su mayor parte a los países de alta industrialización, pero también se lleva a cabo entre países del Tercer Mundo. Hay indicios, además, de que algunos países de industrialización reciente están exportando productos industriales a las economías de planificación central.

A estos cambios deben añadirse las nuevas modalidades y estructuras del comercio de productos energéticos, principalmente el petróleo, con una proporción mayor del valor total del comercio mundial llevada a cabo por los países exportadores de hidrocarburos.

Tales variaciones en las estructuras del comercio han originado también nuevas situaciones en cuanto a poderío económico; esto es, una nueva estructura del poderío económico en que el intercambio basado en tecnología avanzada de los países altamente industrializados es uno de los factores más notables, y el comercio en energéticos, especialmente petróleo y gas de los paises del Oriente Medio y de algunos exportadores de petróleo que no son miembros de la OPEP, es otro ejemplo sobresaliente.

Esta es una situación bastante distinta a la que prevalecía en los años treinta, cuando los países en desarrollo en general estaban en posiciones extremadamente débiles, con relaciones de precios del intercambio fácilmente declinantes y con muy poca capacidad para compensar semejantes descensos de su comercio de productos primarios con mayores exportaciones de manufacturas, Hoy día, inclusive se lleva a cabo comercio de tecnología y servicios entre los países en desarrollo.

Las políticas comerciales a las que están recurriendo todos los países son, como en los años treinta, una vez más, defensivas y proteccionistas. Los países en desarrollo tienen una larga historia de protección arancelaria para los fines de su política de sustitución de importaciones y para el control de su balanza de pagos. No es probable que dicha protección se reduzca hoy día. Los países de alta industrialización que durante algún tiempo, por lo menos durante los años cincuenta y sesenta, mantuvieron políticas bastante abiertas para admitir importaciones a sus mercados en fuerte expansión, están recurriendo ahora a políticas proteccionistas, no sólo entre ellos mismos, sino en contra de los países de nueva industrialización y otros países en desarrollo - con algunas excepciones tales como el sistema de preferencias unilaterales y los beneficios es. peciales que la Comunidad Económica Europea otorga a los países de menor nivel de desarrollo, y otros sistemas similares; pero algu- 
nos de estos esquemas están dejando de funcionar o se están desarticulando.

La situación financiera de los países industrializados se ha dete. riorado internamente, a la par que la situación financiera internacional. Esto es, la dificultad de asegurar la recuperación de los préstamos, y especialmente garantizar los pagos de intereses, no sólo es un problema entre los países desarrollados por un lado, y el resto del mundo por el otro (esto es, incluidos los países socialistas y los países en desarrollo), sino que es también un problema interno en cada país desarrollado en un momento de grave recesión, con tasas reales de interés muy elevadas $y$ muy poca nueva inversión. Desde luego que se ha producido un cambio muy grande en la estructura de la oferta financiera internacional debido a la muy limitada capacidad de los organismos multilaterales para absorber o capturar los petrodólares y los eurodólares creados después de 1971. Ảsí, la proporción de préstamos a largo plazo y a baja tasa de interés y de transferencias blandas o "concesionales" de recursos a favor de los países en desarrollo se ha reducido en relación con el flujo total de fondos. La cooperación económica internacional se ha reducido también, como proporción del PIB de los países industrializados en general, si bien uno o dos países han podido cumplir sus metas de acuerdo con los Decenios del Desarrollo de las Naciones Unidas.

3. Ahora bien, ¿cuál es la naturaleza de la recuperación actual? Es importante examinarla y compararla con las recuperaciones ocurridas en algunas de las fluctuaciones cíclicas pasadas, sobre todo en la depresión de los años treinta. La actual recuperación de la econamía norteamericana, a base de lo que muestran la mayor parte de los indicadores, presenta todavía características de gran incertidumbre y fragilidad, porque, como lo han señalado algunos economistas de los Estados Unidos, este país no constituye ya una economia que pueda funcionar en forma aislada de otras importantes, sobre todo las de Europa Occidental y Japón. En algunos casos se sostiene inclusive que la prosperidad de los países en desarrollo sería un factor en las posibilidades de recuperación firme de la economía norteamericana. Las diferencias esenciales en la recuperación actual parecen ser, primero, que no es muy intensa; segundo, que está ocurriendo con tasas reales de interés muy elevadas a pesar del descenso reciente cle las tasas de interés nominales; tercero, que está expuesta a grandes movimientos incontrolados de capital y a grandes fluctuaciones de los tipos de cambio; y cuarto, que está llevándose a cabo en un ambiente de problemas estructurales más difíciles que en los años treinta. La mayor parte de los analistas de la situación actual y previsible parecen estar de acuerdo en que todo esto quiere decir que el crecimiento económico en los países industrializados de economía de mercado - Estados Unidos, Europa Occidental, Japón y Australia - será lento, esto es, del orden 
Victor L. Utquidi / La actual depresión internacional y los pafses en vias...

del 2 al $3 \%$ anual en comparación con un 4 al $5 \%$ en los años cincuenta y sesenta.

4. La siguiente interrogante es: esta recuperación lenta e incierta, y en general el escaso crecimiento previsible, ¿cómo repercutirá en los países en desarrollo? Suponiendo que haya un efecto inmediato en el fortalecimiento de los precios de algunos productos básicos en los mercados mundiales, y que se produzca una leve apertura para las exportaciones de manufacturas provenientes de los países en desarrollo, sobre todo de los de nueva industrialización, debe reconocerse que los países en desarrollo no podrán por esos solos medios resolver algunos de los problemas básicos antes mencionados. Estos problemas requieren mucha más atención en relación con estructuras, $y$ en tanto puedan resolverse con la ayuda de financiamiento internacional requerirán empréstitos a largo plazo, a baja tasa de interés, y no financiamientos a corto o a mediano plazo a elevadas tasas de interés como los que el sistema bancario internacional ha estado ofreciendo en los últimos años. A esto habrá que añadir los problemas intensos de liquidez en divisas que algunos países en desarrollo han estado experimentando últimamente, y que han hecho necesaria la reestructuración de su deuda externa en medida muy importante. Ciertos países se han puesto técnicamente en estado de moratoria sobre su deuda externa en la medida que no han podido hacer frente a los pagos de intereses, o éstos han tenido que aplazarse. Si los efectos de la recuperación no fueran muy importantes en función de precios, de comercio y manufacturas de los países en desarrollo y de formas menos onerosas de financiamiento, entonces hay que preguntarse: ¿cuáles serían las opciones para los países en desarrollo?

5. Uno de los escenarios probables es que los países altamente industrializados, debido a los problemas que están afrontando, probablemente mirarán hacia adentro y verán con cierta desconfianza los intentos de ayudar a resolver la situación de los países en desarrollo. Tienen bastantes problemas propios y entre sí con qué lidiar, que determinan políticas restrictivas, sobre todo en comercio y finanzas, de manera que será menos probable que presten al mundo en desarrollo la clase de atención que el Informe Brandt y el reciente memorándum Brandt han estado recomendando.

6. Si esta es la situación, junto con el lento crecimiento de los países desarrollados, ¿cuál sería el escenario para los países en desarrollo? En esto deberá recordarse, como antes, que hay diferentes clases de países en desarrollo y que, en consecuencia, algunos podrán hacerle frente mucho mejor que otros a la perspectiva actual e inmediata, y que unos cuantos podrán llevar a cabo su próxima etapa de desarrollo en mejores condiciones que el resto. El resultado neto de las limitaciones al intercambio internacional será, desde luego, la necesidad de formular y clesarrollar políticas más raciona- 
les y conscientes de autovalimiento (self-reliance) en los paises en desarrollo, por lo menos durante los próximos años. Sería casi inconcebible que aún los países en desarrollo de mayor fortaleza económica fueran a abrir sus mercados o a mantenerlos abiertos a las importaciones de los países industrializados, o que fueran a perseverar en su dependencia respecto a préstamos onerosos a corto plazo. Tendrán que adoptar permanentemente un fuerte control de importaciones con sus consecuentes políticas de sustitución de importaciones, como también tendrán que poner en práctica políticas más agresivas de exportación; buscarán obtener alguna forma de relativa estabilidad de precios de sus productos de exportación, y penetrar en los mercados de los páses desarrollados con sus manufacturas. No obstante el éxito aparente del desarrollo de exportaciones de manufacturas de algunos páses de Asia Oriental en los últimos quince años, esto quizá no sea tan fácil en el futuro, y la posición de cada país de nueva industrialización frente a los demás será mucho más competitiva que antes.

7. Un mayor autovalimiento en los principales países en desarrollo irá acompañado probablemente de crecientes esfuerzos de cooperacion "sur-sur", sobre lo cual se ha dicho mucho en los últimos años, pero se ha hecho muy poco. Dicha cooperación puede llevarse a cabo mediante convenios de intercambio complementario, mediante asociaciones de comercio preferencial y mercados comunes (aunque la experiencia de todos estos arreglos no ha tenido mucho exito hasta ahora), así como en materia de inversión, flujos financieros y tecnología. Ha habido bastantes ejemplos de bancos regionales, así como de fondos especiales establecidos por los países petroleros. Son también numerosos los casos de intercambio de servicios técnicos y de resultados de la investigación tecnológica. No obstante, hasta ahora la cooperación sur-sur ha sido bastante limitada, y no se ha generalizado entre los países en desarrollo. Ni siquiera se ha estudiado adecuadamente su potencial ni traducido en los convenios y arreglos necesarios. Los países en desarrollo tien. den todavía a mirar hacia el norte para las soluciones a sus problemas a través del comercio, la inversión, las finanzas o la tecnología. En suma, habrá necesidad de obtener ventajas adicionales en las relaciones sur-norte y de desarrollar simultáneamente posibilidades de cooperación sur-sur. (Padrá suponerse también que la cooperación este-sur no será muy significativa en la perspectiva a corto y a mediano plazo, salvo sobre bases muy selectivas).

8. Debido al crecimiento demográfico rápido de los países en desarrollo, que es todavía de un $2 \%$ al año -no obstante el descenso reciente de las tasas demográficas en algunas áreas-, la perspectiva de que se logren fuertes incrementos del ingreso per cápita no parece ser muy buena para los próximos diez años. Algunos pocos países en desarrollo pudieran encontrarse con excedentes de divisas. 
Unos cuantos tendrán más éxito que otros en la sustitución de importaciones o en la incorporación de tecnología útil no sólo para el mercado interno sino para exportar a los mercados mundiales. Otros podrán persistir en tratar de seguir caminos alternativos de desarrollo, esto es, industrias de baja intensidad de capital en lugar de industrias de alta tecnología y de alta intensidad energética, o de alta intensidad de capital. Algunos tal vez puedan resolver sus problemas agrícolas, sobre todo en cuanto a la producción de alimentos. Otros más podrán llevar a cabo con éxito políticas comerciales. Pero parece haber una limitación general en las posibilidades que se presentan a los países en desarrollo debido a que la mayor parte de ellos necesitan incorporar el alto costo de la energía en un sistema realista de precios. Hay que tener en cuenta también costos adicionales del desarrollo, tales como los que imponen la contaminación ambiental y la degradación de recursos, así como la urbanización demasiado rápida.

9. Muchos países en desarrollo se están movilizando además hacia sistemas de bienestar social similares a los de los países de alta industrialización, con la necesidad adicional de establecer coberturas incrementadas en sus programas gratuitos de salud y educación. Todo esto ante sistemas fiscales y tributarios bastante débiles. Algunos países en desarrollo están empezando a dedicar una proporción elevada de su PrB y de su presupuesto a gastos militares, con frecuencia para adquirir material que se vuelve obsoleto a corto plazo, y que tiene que ser reemplazado por importaciones aún más caras de dicho tipo. Esta es una evidente desviación de recursos hacia objetivos no productivos, que en la mayor parte de los casos ni siquiera genera alguna consecuencia útil al sector civil de la economía que pudiera derivarse de la investigación tecnológica.

10. Para concluir este breve examen de situación, debe recalcarse que la mera recuperación de las economías de los países altamente industrializados - que tendrá en todo caso miras fuertemente internas- no traerá probablemente mucho alivio ni beneficio al mundo en desarrollo, y que la perspectiva de crecimiento lento de los países de economfa de mercado industriales hará necesario probablemente que los países en desarrollo reevalúen sus perspectivas inmediatas y de plazo intermedio, y lleven a cabo un esfuerzo mucho más claro e intenso de autovalimiento, individual y colectivamente. Esto, desde luego, será para bien de ellos mismos. 